\title{
Evidence for Covalent Linkages between Chitin and $\beta$-Glucan in a Fungal Wall
}

\author{
By J. H. SIETSMA AND J. G. H. WESSELS \\ Department of Developmental Plant Biology, Biological Centre, \\ University of Groningen, Haren, The Netherlands
}

(Received 6 October 1978)

\begin{abstract}
Covalent linkages between chitin and $\beta$-glucan in the wall of Schizophyllum commune were indicated by the markedly changed solubility characteristics of the glucan when chitin was specifically removed either (i) by enzymic digestion with purified chitinase or (ii) by first deacetylating the chitin with alkali followed by depolymerization of the deacetylated chitin with nitrous acid. After depolymerization of the chitin, two types of $\beta$-glucans could be isolated: one was water-soluble and highly branched, the other was alkali-soluble with branches only one glucose unit long. Lysine $(50 \%)$ and citrulline $(20 \%)$ were the major amino acids in the R-glucan/chitin complex. By digesting $90 \%$ of the $\beta$-glucan in the R-glucan/chitin complex with $(1 \rightarrow 3)$ - $\beta$-glucanase, a residue was obtained which, on hydrolysis with chitinase, yielded $N$-acetylglucosamine and a compound containing ( $N$-acetyl)glucosamine, lysine and/or citrulline. A model is proposed for the R-glucan/chitin complex in which amino acids, especially lysine and citrulline, are involved in the linkages between glucan and chitin.
\end{abstract}

\section{INTRODUCTION}

The alkali-insoluble portion of the hyphal wall of most fungi, except Phycomycetes, appears to contain chitin microfibrils embedded in a matrix of R-glucan, which mainly consists of $(1 \rightarrow 3)$ - and $(1 \rightarrow 6)$-linked $\beta$-glucan (for a review, see Rosenberger, 1976). The results of a detailed study of wall architecture in the basidiomycete Schizophyllum commune were consistent with this view (van der Valk et al., 1977) but the results of a chemical analysis of the structure of R-glucan did not explain the extreme insolubility of the glucan (Sietsma \& Wessels, 1977). Therefore, covalent linkages between the highly insoluble chitin and R-glucan were proposed. The present study was aimed at obtaining more evidence for the existence of such linkages. The results indicate that a large proportion of the glucosamine residues in chitin are linked to $\beta$-glucan via amino acids, particularly lysine and citrulline.

\section{METHODS}

Preparation of the $R$-glucan/chitin complex. This was isolated as the alkali-resistant residue from the walls of Schizophyllum commune strain 1-40 ( = 699), as described previously (Sietsma \& Wessels, 1977). R-glucan/ chitin labelled with ${ }^{14} \mathrm{C}$ was isolated from mycelium cultivated in minimal medium supplemented with $0 \cdot 1 \mathrm{mCi}\left[\mathrm{U}-{ }^{14} \mathrm{C}\right] \mathrm{glucose}$ per $100 \mathrm{ml}$ medium.

Isolation and purification of enzymes. Chitinase was produced by cultivating a strain of Streptomyces satsumaensis in a medium with colloidal chitin as a carbon source (containing, per litre, $5 \mathrm{~g}$ chitin, $0.5 \mathrm{~g}$ yeast extract, $2 \mathrm{~g} \mathrm{KH}_{2} \mathrm{PO}_{4}, 0.5 \mathrm{~g} \mathrm{KCl}, 1 \mathrm{~g} \mathrm{MgSO}_{4}, 1 \mathrm{~g}$ peptone, $0.5 \mathrm{~g} \mathrm{NaCl}$ and $1 \mathrm{~g} \mathrm{NaNO} ; \mathrm{pH} \mathrm{6.5)} \mathrm{for} 5 \mathrm{~d}$ at $25^{\circ} \mathrm{C}$ on a rotary shaker. The enzyme was isolated from the culture filtrate by adding 2 vol. acetone at $-30^{\circ} \mathrm{C}$. The precipitate was suspended in water and dialysed against $0.005 \mathrm{M}$-acetate buffer $\mathrm{pH} 5.8$ for $24 \mathrm{~h}$. After centrifugation to remove insoluble material, the supernatant was freeze-dried yielding the crude chitinase preparation. The chitinase was purified by adsorbing it twice on colloidal chitin as described by 
Jeuniaux (1966) and further by gel filtration on Sephadex G-100. Fractions containing the purified chitinase were pooled and freeze-dried. Exo- $(1 \rightarrow 3)-\beta$-glucanase was isolated and characterized as described before (Sietsma \& Wessels, 1977).

Assay of enzyme activities. The activities of $(1 \rightarrow 3)-\beta$-glucanase, $(1 \rightarrow 6)-\beta$-glucanase and chitinase were measured by incubation with, respectively, the $(1 \rightarrow 3)-\beta$-glucan laminaran (Koch-Light), the $(1 \rightarrow 6)-\beta$ glucan pustulan (Calbiochem) and colloidal chitin (crustacean chitin; Fluka; purified according to the procedure of Skujins et al., 1965) at a concentration of $1 \mathrm{mg} \mathrm{ml}^{-1}$ in $0.05 \mathrm{M}$-sodium acetate buffer $\mathrm{pH} 5.8$ at $35^{\circ} \mathrm{C}$ for $1 \mathrm{~h}$. The increase in reducing groups (as glucose equivalents) was determined by the neocuproine method (Dygert et al., 1965). An enzyme unit is defined as that amount of enzyme releasing $1 \mu$ mol glucose equivalents $\mathrm{h}^{-1}$ at $35^{\circ} \mathrm{C}$.

Proteolytic activity was measured on Azocoll (Calbiochem).

Enzymic treatments. R-glucan/chitin was incubated with purified chitinase at $35^{\circ} \mathrm{C}$ in acetate buffer pH 5.8 with toluene added as bacterial inhibitor. Other enzymic treatment of the R-glucan/chitin complex was done as described by Sietsma \& Wessels (1977).

Nitrous acid treatment. To $10 \mathrm{mg}$ material suspended in $1 \mathrm{ml}$ water in a screw-capped tube, $1.5 \mathrm{ml}$ freshly prepared $2 \mathrm{M}-\mathrm{NaNO}_{2}$ solution was added followed by $0.5 \mathrm{ml} 2 \mathrm{M}-\mathrm{HCl}$, giving a pH of 3, and the tube was then closed. After $1.5 \mathrm{~h}$ at room temperature the tube was opened and filtered air was bubbled through the suspension for $30 \mathrm{~min}$. The insoluble residue was washed three times with water and a portion was used for determining the total amount of carbohydrate. The rest was treated with $1 \mathrm{M}-\mathrm{KOH}$ under $\mathrm{N}_{2}$ at $60^{\circ} \mathrm{C}$ for $20 \mathrm{~min}$ and the amount of carbohydrate in the insoluble residue was determined. The R-glucan/chitin complex was either treated directly with nitrous acid or the chitin in the complex was first deacetylated by treatment with $40 \%(\mathrm{w} / \mathrm{v}) \mathrm{NaOH}$ for $1 \mathrm{~h}$ at $100{ }^{\circ} \mathrm{C}$ under $\mathrm{N}_{2}$ (Horton \& Lineback, 1965). The nitrous acidsoluble products were separated on columns of Sephadex G-10 and G-50, hydrolysed by chemical ( $1 \mathrm{M}-\mathrm{HCl}$ at $100{ }^{\circ} \mathrm{C}$ for $1 \mathrm{~h}$ ) and enzymic [exo-( $(1 \rightarrow 3)-\beta$-glucanase at $35^{\circ} \mathrm{C}$ in acetate buffer $\mathrm{pH} 5.8$ for $16 \mathrm{~h}$ ] treatments and the hydrolysis products were identified by thin-layer chromatography. The nitrous acid-insoluble product was treated with exo-( $1 \rightarrow 3)-\beta$-glucanase and the solubilized products were also identified by thinlayer chromatography.

Amino acids analysis. The R-glucan/chitin complex was divided into an acid-soluble fraction ( $0.5 \mathrm{M}-\mathrm{HCl}$, $100^{\circ} \mathrm{C}, 1 \mathrm{~h}$ ) and an insoluble residue (see Sietsma \& Wessels, 1977). Both fractions were hydrolysed with $0.25 \mathrm{M}-\mathrm{HCl}$ in the presence of Dowex $50 \mathrm{~W}-\mathrm{X} 8$ in the $\mathrm{H}^{+}$form at $100{ }^{\circ} \mathrm{C}$ for $24 \mathrm{~h}$. The Dowex was eluted with $2 \mathrm{M}-\mathrm{HCl}$ and the eluate was concentrated under reduced pressure; the amino acids were then analysed with a Beckman Unichrom amino acid analyser, equipped with a two-column system. The acid and neutral amino acids were separated on a spherical ion-exchange resin type M72 and the basic amino acids on a spherical ion-exchange resin type M71 (supplied by Beckman).

Other methods. Total carbohydrate content was determined with anthrone (Fairbain, 1953). Free amino groups were estimated with the ninhydrin reagent (Spies, 1957). Chitin was determined by first removing the glucan by successive treatments with dilute acid and alkali (Sietsma \& Wessels, 1977), then hydrolysing the residue with $6 \mathrm{M}-\mathrm{HCl}$ at $120^{\circ} \mathrm{C}$ for $2 \mathrm{~h}$ under $\mathrm{N}_{2}$ and measuring the amount of glucosamine in the hydrolysate according to Johnson (1971). Thin-layer chromatography was done on cellulose plates with the following solvent systems: (a) butan-1-ol/ethanol/water (3:1:1, by vol.); (b) ethyl acetate/pyridine/acetic acid/water $(5: 5: 1: 3$, by vol.). Radioactivity on the chromatograms was measured with a Berthold thin-layer scanner. Reducing compounds were detected with the aniline phthalate reagent, compounds with free amino groups with the ninhydrin reagent and poly-alcohols with alkaline $\mathrm{AgNO}_{3}$. For column chromatography, Sephadex G-10 and G-50 (Pharmacia) were swollen and equilibrated in 0.005 M-sodium acetate buffer pH 5.8.

\section{RESULTS}

\section{Purification of chitinase}

The crude chitinase preparation isolated from the culture filtrate of Streptomyces satsumaensis contained (per mg dry wt) 951 units of chitinase, but it also showed activity towards laminaran (566 units) and pustulan (463 units). The latter substrates contain linkages also present in R-glucan (Sietsma \& Wessels, 1977) and therefore these activities had to be removed. After adsorption to chitin and gel permeation chromatography (see Methods), the ratio of the activities towards chitin, laminaran and pustulan became 100:0 006:0.002. No protease was detected in any of the enzyme preparations. 


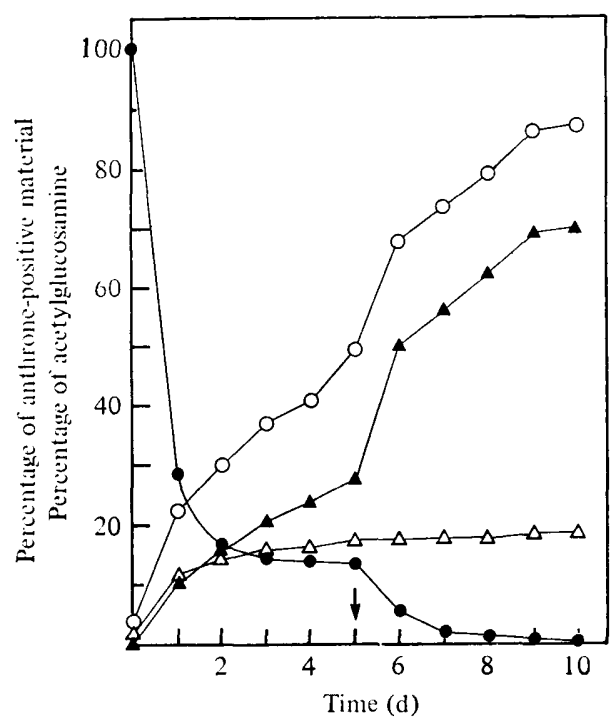

Fig. 1. Effect of purified chitinase on the solubility characteristics of R-glucan. R-glucan/chitin $(10 \mathrm{mg})$ was incubated with purified chitinase $(0.25 \mathrm{mg})$ in $0.05 \mathrm{M}$-acetate buffer $\mathrm{pH} 5.8$ at $35^{\circ} \mathrm{C}$ : , percentage of chitin remaining in the insoluble residue; $O$, percentage of total glucan solubilized; $\Delta$, percentage of anthrone-positive material soluble in water; $\Delta$, percentage of anthrone-positive material soluble in $1 \mathrm{M}-\mathrm{KOH}$. The arrow indicates the time at which the insoluble residue was sedimented and fresh enzyme was added.

Table 1. Effect of several consecutive treatments on the solubility of the glucan from the R-glucan/chitin complex

Treatment

1. $40 \%(w / v) ~ N a O H, 1 ~ h, 100{ }^{\circ} \mathrm{C}$

2. Treatment 1 , followed by nitrous acid

3. Treatment 2 , followed by $1 \mathrm{M}-\mathrm{KOH}, 20 \mathrm{~min}, 60^{\circ} \mathrm{C}$

4. $\mathrm{H}_{2} \mathrm{O}, 1 \mathrm{~h}, 100^{\circ} \mathrm{C}$

5. Treatment 4 , followed by nitrous acid

6. Treatment 5 , followed by $1 \mathrm{M}-\mathrm{KOH}, 20 \mathrm{~min}, 60^{\circ} \mathrm{C}$

7. Pronase, $20 \mathrm{~h}, 30^{\circ} \mathrm{C}$, pH $7 \cdot 2$

8. Treatment 7 , followed by nitrous acid

9. Treatment 8 , followed by $1 \mathrm{M}-\mathrm{KOH}, 20 \mathrm{~min}, 60^{\circ} \mathrm{C}$
Percentage of anthrone-positive material remaining insoluble
$98 \cdot 4$
$40 \cdot 7$
$4 \cdot 6$
$97 \cdot 6$
$98 \cdot 6$
$56 \cdot 8$
$97 \cdot 8$
$98 \cdot 6$
$56 \cdot 4$

\section{Effect of purified chitinase on the R-glucan/chitin complex}

The alkali-resistant part of the hyphal wall was treated with the purified chitinase preparation, and the changes in the amounts of both the water-soluble and alkali-soluble anthrone-positive material and in the amount of chitin were monitored over a $5 \mathrm{~d}$ period. After $5 \mathrm{~d}$, the insoluble material was sedimented, fresh enzyme was added and the experiment was continued for another $5 \mathrm{~d}$. The results (Fig. 1) show that the purified chitinase, by digesting the chitin, rendered most of the anthrone-positive material from the R-glucan/ chitin complex soluble in water, with the rest of the glucan becoming almost completely soluble in $1 \mathrm{M}-\mathrm{KOH}$. Laminaran and pustulan incubated with the same enzyme preparation for $10 \mathrm{~d}$ were only hydrolysed $4 \cdot 1$ and $0 \cdot 2 \%$, respectively. If the effects of purified pustulanase and laminaranase from $S$. commune on the R-glucan/chitin complex (Wessels, 1969) are taken into account, it follows that the low activities present in the purified chitinase preparation could be responsible for only 0.7 and $0.4 \%$ of the liberated glucan, respectively. 


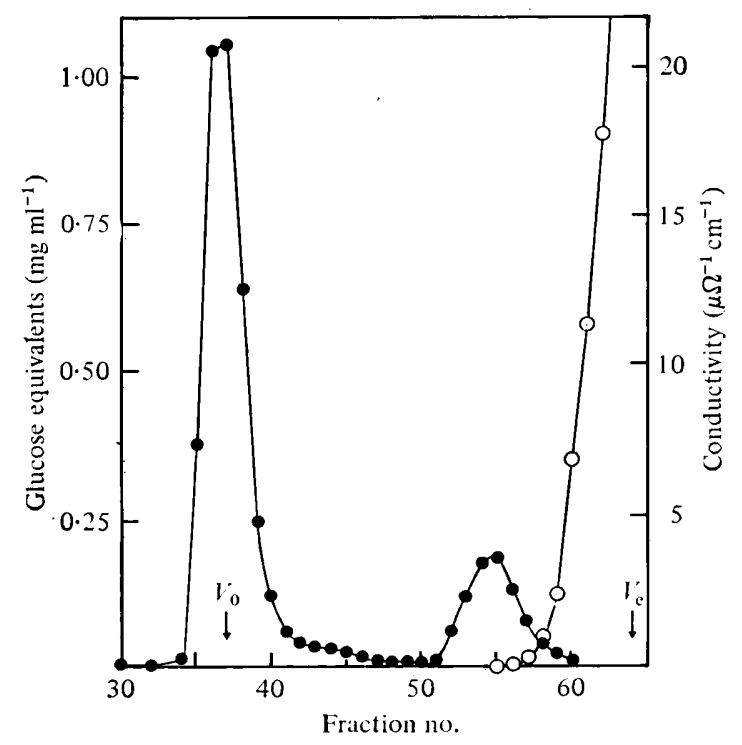

Fig. 2. Column chromatography on Sephadex G-10 of the soluble products obtained after treatment of R-glucan/chitin (100 mg; previously deacetylated by treatment with $40 \% \mathrm{NaOH}, 1 \mathrm{~h}, 100^{\circ} \mathrm{C}$ ) with nitrous acid as described in Methods. The column was eluted with $0.005 \mathrm{M}$-acetate buffer pH $5.8 ; V_{0}$, void volume; $V_{\dot{e}}$, end volume. The fractions were analysed with the anthrone reagent $(\odot)$ and their conductivity $(O)$ was measured.

\section{Effect of nitrous acid}

Treatment with nitrous acid causes depolymerization of 2-amino-2-deoxyglycans but has no effect on $N$-acetyl-2-amino-2-deoxyglycans (Shively \& Conrad, 1970; Horton \& Philips, 1973; Datema et al., 1977). Thus, if chitin is first deacetylated, nitrous acid should cause complete depolymerization. Table 1 shows that when the chitin in the R-glucan/chitin complex was first deacetylated with strong alkali $\left(40 \% \mathrm{NaOH}, 1 \mathrm{~h}, 100{ }^{\circ} \mathrm{C}\right)$, nitrous acid solubilized half of the glucan and made the rest alkali-soluble. Table 1 further shows that R-glucan/chitin treated with hot water also contained some nitrous acid-sensitive groups, but in this case only part of the glucan became alkali-soluble. Pronase treatment apparently did not generate nitrous acid-sensitive groups, nor did it release any glucan by itself. This emphasizes that proteins were probably not involved in keeping the glucan insoluble.

To investigate whether nitrous acid-sensitive groups were generated by the preparation procedure for the R-glucan/chitin complex [in which S-glucan, i.e. $(1 \rightarrow 3)$ - $\alpha$-glucan, is removed by treating the wall with $1 \mathrm{M}-\mathrm{KOH}$ for $20 \mathrm{~min}$ at $60^{\circ} \mathrm{C}$ ], complete walls were treated with nitrous acid. This treatment caused the release by alkali of $5.5 \%$ of the total glucan (i.e. about $10 \%$ of the glucan in the R-glucan/chitin complex), in addition to the solubilized S-glucan. Thus it appears that complete walls did contain some non-acetylated hexosamine residues but that treatment of the walls with $1 \mathrm{M}-\mathrm{KOH}$ at $60{ }^{\circ} \mathrm{C}$ to remove S-glucan generated additional nitrous acid-sensitive sites (Table 1).

\section{Analysis of the products of nitrous acid treatment of the deacetylated $R$-glucan/chitin complex}

When R-glucan/chitin was first deacetylated and subsequently treated with nitrous acid, the chitin was depolymerized and $59.3 \%$ of the glucan was solubilized (Table 1). The residual material completely dissolved in alkali showing that no chitin remained. The nitrous acid-soluble material was separated into high molecular weight and low molecular weight anthrone-positive components by chromatography on Sephadex G-10 (Fig. 2). The 
Table 2. Mole fractions of amino acids in the alkali-insoluble portion of the hyphal wall of Schizophyllum commune

\begin{tabular}{lccc} 
Amino acid & \multicolumn{2}{c}{ Fraction* } & Total \\
Lysine & Acid-insoluble & Acid-soluble & 0.485 \\
Citrulline & 0.495 & 0.483 & 0.205 \\
Glutamic acid & 0.131 & 0.224 & 0.127 \\
Threonine & 0.060 & 0.145 & 0.047 \\
Glycine & 0.045 & 0.048 & 0.036 \\
Alanine & 0.089 & 0.023 & 0.024 \\
Aspartic acid & 0.059 & 0.015 & 0.020 \\
Serine & 0.044 & 0.014 & 0.019 \\
Valine & 0.059 & 0.009 & 0.017 \\
Leucine & 0.020 & 0.016 & 0.013 \\
Isoleucine & trace & 0.016 & 0.006
\end{tabular}

* The acid-insoluble and acid-soluble fractions contain 19 and $81 \%$, respectively, of the total amino acids (Sietsma \& Wessels, 1977).

high molecular weight component, representing $53.2 \%$ of the total anthrone-positive material of the R-glucan, was rechromatographed on Sephadex G-50 and again accumulated in the void volume of the column, indicating that its molecular weight was higher than 10000 . Complete enzymic hydrolysis with exo- $(1 \rightarrow 3)-\beta$-glucanase followed by thin-layer chromatography revealed glucose, gentiobiose and higher $(1 \rightarrow 6)$ - $\beta$-linked oligosaccharides as products. The high molecular weight fraction therefore consisted of a highly branched $(1 \rightarrow 3)$ - $\beta$-glucan with side branches of different lengths containing $(1 \rightarrow 6)-\beta$-linkages (see Sietsma \& Wessels, 1977). The low molecular weight fraction, which represented $5 \cdot 6 \%$ of the total anthrone-positive material of the R-glucan, eluted from Sephadex G-10 in the same region as disaccharides (cellobiose and maltose). Thin-layer chromatography of this fraction revealed a spot $\left(R_{\text {Glc }} 0.52\right.$ in solvent system b) reacting positively with alkaline $\mathrm{AgNO}_{3}$, as well as traces of $\mathrm{N}$-acetylglucosamine and anhydromannose. The latter two substances are products of the reaction of partly deacetylated chitin with nitrous acid; the bulk of these substances eluted together with the salt in the end volume of the column. Hydrolysis of the material with an $R_{\mathrm{Glc}}$ of 0.52 with $6 \mathrm{M}-\mathrm{HCl}$ for $2 \mathrm{~h}$ at $120^{\circ} \mathrm{C}$ followed by thin-layer chromatography showed glucose, anhydromannose and glucosamine, indicating that this material was a compound containing glucose, anhydromannose derived from glucosamine and/or $\mathrm{N}$-acetylglucosamine (incomplete deacetylation by alkali).

The nitrous acid-insoluble fraction, accounting for $40.7 \%$ of the total anthrone-positive material of the R-glucan, dissolved readily in $1 \mathrm{M}-\mathrm{KOH}$. It was also completely solubilized when treated with exo- $(1 \rightarrow 3)-\beta$-glucanase at $\mathrm{pH} 5 \cdot 8$. The reaction products, as revealed by thin-layer chromatography, were only glucose and gentiobiose. Thus, this fraction contained a $(1 \rightarrow 3)$ - $\beta$-glucan with branches of single glucose units attached by $(1 \rightarrow 6)-\beta$-linkages, resembling the glucan excreted into the medium by this organism (see Wessels et al., 1972; Sietsma \& Wessels, 1977).

\section{Amino acids in the $R$-glucan/chitin complex}

The R-glucan/chitin complex contained $10.7 \%$ amino acids, $81.2 \%$ of which can be solubilized by $0.5 \mathrm{M}-\mathrm{HCl}$ at $100{ }^{\circ} \mathrm{C}$ (Sietsma \& Wessels, 1977). Analysis of these amino acids revealed that lysine, citrulline and glutamic acid accounted for more than $80 \%$ of the amino acids, lysine alone accounting for nearly $50 \%$ (Table 2 ).

To investigate the possibility that these amino acids were involved in the linkage between chitin and R-glucan, the R-glucan/chitin complex was treated extensively with exo-( $1 \rightarrow 3)$ $\beta$-glucanase to dissolve most of the glucan. The insoluble residue was then analysed for 
Table 3. Composition of the residue remaining after exo-( $1 \rightarrow 3)-\beta$-glucanase treatment of $R$-glucan/chitin compared with the composition of the original $R$-glucan/chitin complex from Schizophyllum commune

Composition as a percentage of the dry weight of the original R-glucan/chitin

\begin{tabular}{|c|c|c|c|c|}
\hline & \multicolumn{4}{|c|}{$\begin{array}{c}\text { Composition as a percentage of the dry weight of } \\
\text { the original R-glucan/chitin }\end{array}$} \\
\hline & \multicolumn{2}{|c|}{$\begin{array}{l}\text { Residue after exo- }(1 \rightarrow 3)-\beta- \\
\text { glucanase treatment }\end{array}$} & \multicolumn{2}{|c|}{ Original R-glucan/chitin } \\
\hline Component & HCl-soluble & $\begin{array}{l}\text { Insoluble } \\
\text { residue }\end{array}$ & HCl-soluble & $\begin{array}{l}\text { Insoluble } \\
\text { residue }\end{array}$ \\
\hline lucose $\left(-\mathrm{H}_{2} \mathrm{O}\right)^{*}$ & $5 \cdot 7$ & - & $56 \cdot 7$ & - \\
\hline Acetylglucosamine $\dagger$ & $0 \cdot 8$ & $17 \cdot 8$ & $5 \cdot 5$ & $17 \cdot 7$ \\
\hline Amino acids $\ddagger$ & $7 \cdot 1$ & $2 \cdot 2$ & $7 \cdot 3$ & $2 \cdot 3$ \\
\hline
\end{tabular}

* Determined as anthrone-positive material in unhydrolysed samples.

$\dagger$ Determined as glucosamine in samples hydrolysed with $6 \mathrm{M}-\mathrm{HCl}$.

¥ Determined as ninhydrin-positive material (leucine as standard) in samples hydrolysed with $6 \mathrm{M}-\mathrm{HCl}$ after correction for the ninhydrin value of glucosamine.

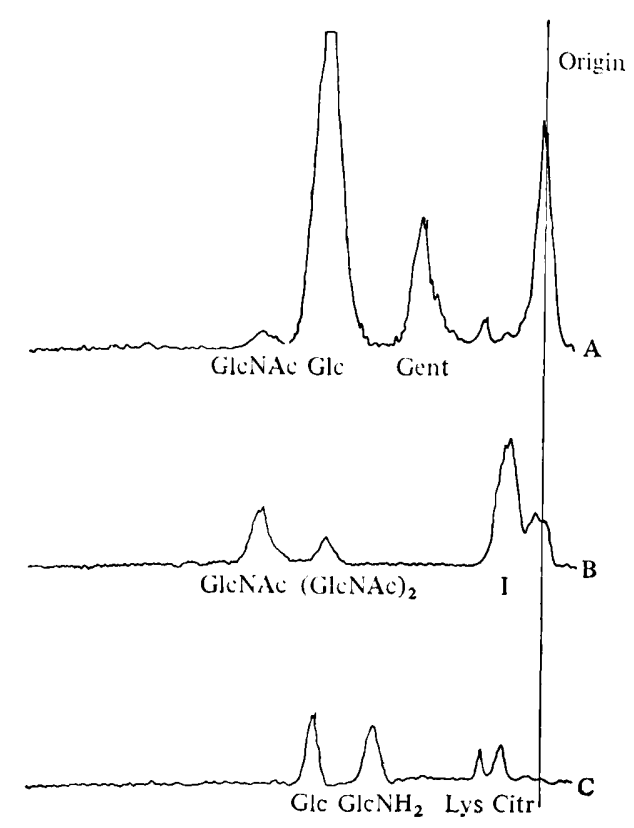

Fig. 3. Radiochromatographic tracings of thin-layer chromatograms in solvent system b: (A) the solubilized products after treating ${ }^{14} \mathrm{C}$-labelled R-glucan/chitin $\left(5 \cdot 2 \mathrm{mg}\right.$ glucose equivalents $\mathrm{ml}^{-1}$; 989500 c.p.m. $\left.\mathrm{ml}^{-1}\right)$ with exo- $(1 \rightarrow 3)-\beta$-glucanase $\left(0.6\right.$ units $\left.\mathrm{ml}^{-1}\right)$ in acetate buffer $\mathrm{pH} 5.8$ at $35{ }^{\circ} \mathrm{C}$ for $48 \mathrm{~h}$; (B) the products of digesting the insoluble residue remaining after treatment $\mathrm{A}$ with chitinase $\left(0.5 \mathrm{mg} \mathrm{ml}^{-1}\right.$ in acetate buffer $\mathrm{pH} 5.8$, at $35^{\circ} \mathrm{C}$ for $\left.24 \mathrm{~h}\right) ;(\mathrm{C})$ the products of hydrolysis of the material in peak I from B with $1 \mathrm{M}-\mathrm{HCl}$ at $100^{\circ} \mathrm{C}$ for $1 \mathrm{~h}$. The components were characterized by co-chromatography with standards. Diacetylchitobiose and gentiobiose were also identified by isolating the spots from the chromatograms, hydrolysing with $6 \mathrm{M}-\mathrm{HCl}$ at $120^{\circ} \mathrm{C}$ for $2 \mathrm{~h}$ and rechromatography; the former gave glucosamine and the latter glucose as products. Abbreviations: GlcNAc, $N$-Acetylglucosamine; (GlcNAc) ${ }_{2}$, di- $N$-acetylchitobiose; Glc, glucose; $\mathrm{GlcNH}_{2}$, glucosamine; Gent, gentiobiose; Lys, lysine; Citr, citrulline. 
glucose, acetylhexosamine and amino acids. The results were compared with the composition of the original R-glucan/chitin complex (Table 3; see also Sietsma \& Wessels, 1977). It appears that the enzymic treatment dissolved $90 \%$ of the acid-soluble glucan, which is the major part of the R-glucan, and most of the non-chitinous (acid-soluble) hexosamine. This is in agreement with earlier findings that $N$-acetylglucosamine is always one of the products of exo-( $1 \rightarrow 3)-\beta$-glucanase treatment of R-glucan/chitin (Sietsma \& Wessels, 1977). The chitin in the R-glucan/chitin complex (i.e. the acid-insoluble hexosamine) and the amino acids were not affected by this enzymic treatment.

The chitin/amino acid complex, containing (acetyl)glucosamine and amino acids in a ratio of approximately $2: 1(\mathrm{w} / \mathrm{w})$, was isolated labelled with ${ }^{14} \mathrm{C}$ by treating radioactive $\mathrm{R}$-glucan/chitin with exo-( $1 \rightarrow 3)-\beta$-glucanase. The main soluble products released by this enzymic treatment were oligosaccharides $[(1 \rightarrow 6)-\beta$-linked], gentiobiose, glucose and $N$ acetylglucosamine (Fig. 3, A), as reported previously (Sietsma \& Wessels, 1977). The ${ }^{14} \mathrm{C}$-labelled insoluble residue was then dissolved by treatment with purified chitinase; the products, separated by thin-layer chromatography (Fig. 3, B), were $N$-acetylglucosamine, di- $N$-acetylchitobiose and an unknown spot (I). The latter was isolated from the chromatogram, hydrolysed with $1 \mathrm{M}-\mathrm{HCl}$ for $1 \mathrm{~h}$ at $100^{\circ} \mathrm{C}$ and rechromatographed; the products ran in positions identical to those of glucose, glucosamine, lysine and citrulline (Fig. 3, C). This does not unequivocally prove that the two amino acids were the products of hydrolysis of a higher molecular weight substance contained in peak I. However, when the material of the chitinase digestion was put on a column of Sephadex G-50 and eluted with water, a discrete peak appeared at an elution volume corresponding to a molecular weight of about 360 . Thin-layer chromatography of the material in this peak showed that it had the same mobility as peak I (Fig. 3, B) and upon hydrolysis it yielded glucosamine, lysine and citrulline. Thus, both lysine and citrulline, the major amino acids in the R-glucan/chitin complex (Table 2), appeared to be linked to chitin and probably involved in the linkage to the $\beta$-glucan.

\section{DISCUSSION}

The present study shows that enzymic or chemical degradation of chitin in the alkaliinsoluble fraction of the wall of Schizophyllum commune causes a marked increase in the solubility of the $\beta$-glucan, indicating the existence of covalent linkages between these two components. The selectivity of the enzymic treatment was substantiated by the purity of the chitinase preparation; hardly any glucanase activity which could have affected the glucan was detectable. Glucosamine-containing polymers were also degraded by a nitrous acid treatment which causes depolymerization by converting glucosaminyl residues to 2,5anhydromannose and simultaneously cleaving the neighbouring glycosidic bond (Shively \& Conrad, 1970; Horton \& Philips, 1973; Datema et al., 1977). The native wall contained few nitrous acid-sensitive sites but the alkali used in the isolation of the R-glucan/chitin complex probably generated more deacetylated glucosamine residues so that subsequent nitrous acid treatment made about $50 \%$ of the glucan soluble in alkali.

A similar effect of nitrous acid on the alkali-insoluble residue of the wall of Aspergillus niger was noted by Stagg \& Feather (1973). Treatment of the R-glucan/chitin complex of $S$. commune with strong alkali at high temperature to deacetylate most of the $N$-acetylglucosamine maximized the effect of nitrous acid, making all the $\beta$-glucan water- or alkalisoluble (Table 1). With respect to the selectivity of this treatment, it should be noted that about $16 \%$ of the $N$-acetylglucosamine of the R-glucan/chitin complex did not occur in chitin and could be solubilized by exo- $(1 \rightarrow 3)-\beta$-glucanase, so that degradation of this part could also have contributed to the solubilization of the glucan.

If covalent linkage between $\beta$-glucan and chitin is accepted, the details of the structure of the R-glucan/chitin complex are best discussed with reference to Fig. 4 which depicts a possible model based on results from the present study and on earlier work. 


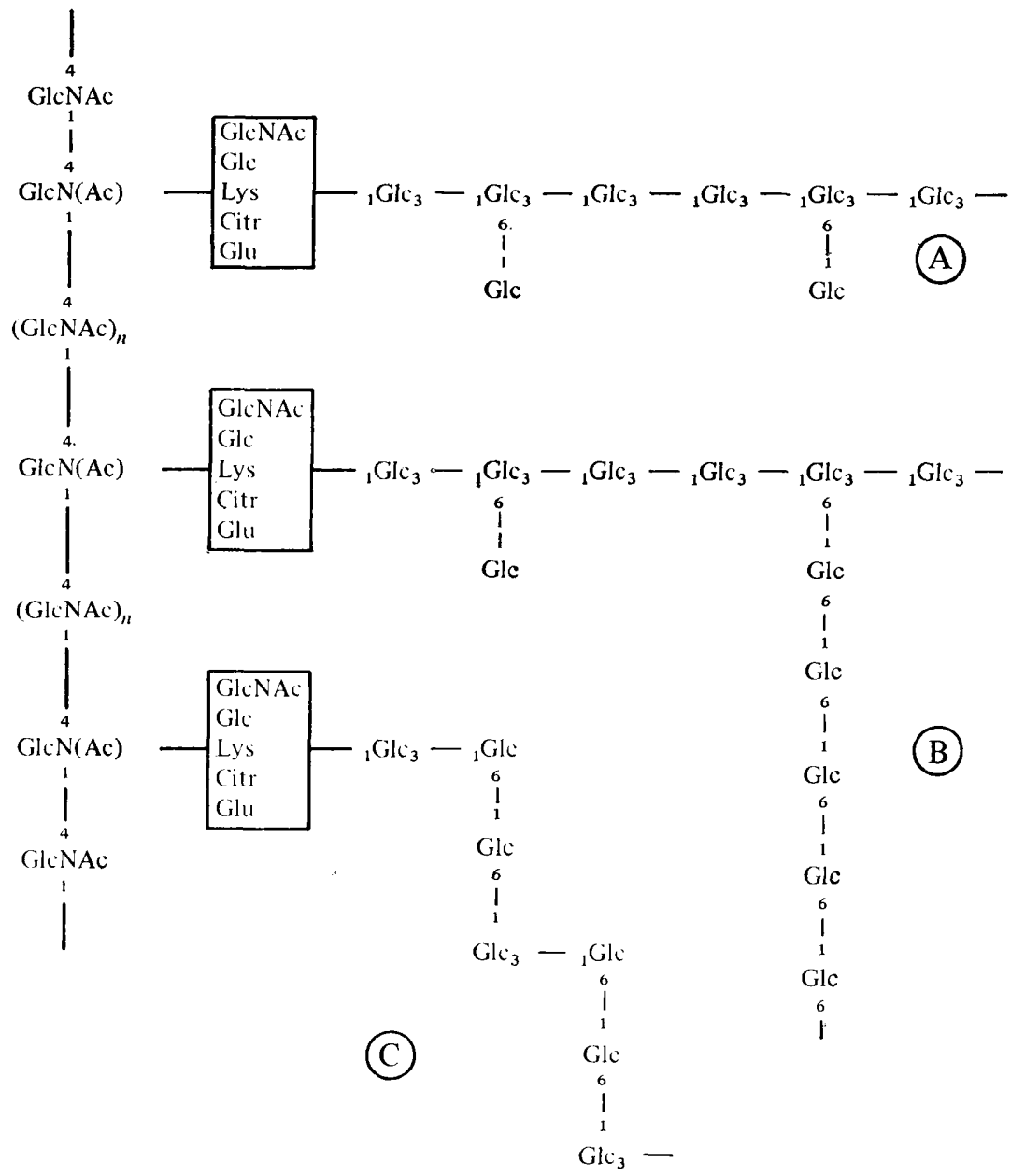

Fig. 4. Proposed model for the structure of the R-glucan/chitin complex of Schizophyllum commune. Glu, Glutamic acid; for other abbreviations, see legend to Fig. 3.

Chitin is defined here as a polymer essentially consisting of $(1 \rightarrow 4)-\beta$-linked $N$-acetylglucosamine that can be hydrolysed by chitinase but not by $(1 \rightarrow 3)-\beta$-glucanase or dilute mineral acid. Only $84 \%$ of the $N$-acetylglucosamine in the R-glucan/chitin complex would then be considered as present in chitin (comprising $18.7 \%$ of the dry weight of the complex); $16 \%$ is released by treatment with either exo- $(1 \rightarrow 3)-\beta$-glucanase or $0.5 \mathrm{M}-\mathrm{HCl}$ at $100{ }^{\circ} \mathrm{C}$ (Sietsma \& Wessels, 1977). However, the model does not suggest that the chitin so defined also occurs as microfibrils of microcrystalline $\alpha$-chitin. In fact, abundant chitin microfibrils and X-ray reflections of crystalline $\alpha$-chitin only become visible after removal of most of the glucan (Sietsma \& Wessels, 1977; van der Valk et al., 1977). Therefore it is possible that the removal of glucan leads to the formation of chitin microfibrils by allowing crystallization of previously non-crystalline chitin chains. Such chitin microfibrils consisting of $\alpha$-chitin can arise spontaneously from non-crystalline chitin (Muzzarelli, 1977). In untreated (freezeetched) preparations, clean chitin microfibrils could only be seen in the case of regenerating protoplasts of $S$. commune which do not synthesize $\beta$-glucan (van der Valk \& Wessels, 1976). It is also significant that polyoxin $D$, a specific inhibitor of chitin synthesis, prevented the formation of the insoluble $\beta$-glucan (de Vries \& Wessels, 1975), probably by preventing the synthesis of an insoluble polyhexosamine to which the $\beta$-glucan could attach. 
The basic structure of the glucan in the R-glucan/chitin complex of $S$. commune was elucidated in an earlier study (Sietsma \& Wessels, 1977). Extensive degradation of the $\mathrm{R}$-glucan with $(1 \rightarrow 3)-\beta$-glucanase resulted in a residue of chitin still containing a small glucan fragment (average degree of polymerization 10). The probable structure of this glucan, comprising $16 \%$ of the total glucan, is depicted as C in Fig. 4 . The present study shows that nitrous acid treatment of the deacetylated R-glucan/chitin complex solubilized the major part of the glucan as two distinguishable species in about equal amounts: one soluble in water (Fig. 4, B) and the other in alkali (Fig. 4, A). In the model these three different variants of $(1 \rightarrow 3)-\beta,(1 \rightarrow 6)-\beta$-linked glucans are depicted as attached separately to the chitin. However, it cannot be excluded that direct bridges between these glucans occur, e.g. involving $\mathrm{N}$-acetylglucosamine. Of special interest is glucan A because it closely resembles a glucan that is excreted into the culture medium in different amounts depending on culture conditions (Wessels et al., 1972; Sietsma et al., 1977; Sietsma \& Wessels, 1977).

Amino acids have commonly been found in the alkali-insoluble glucan/chitin complexes of fungi and have generally been thought to be derived from peptidoglycans (Rosenberger, 1976). The present study discloses the remarkable composition of these amino acids in S. commune. Almost $50 \%$ consisted of lysine, $20 \%$ of citrulline and about $12.5 \%$ of glutamic acid. An abundance of lysine in certain fractions of the alkali-insoluble part of fungal walls has been reported previously, e.g. in Verticillium albo-atrum (Wang \& BartnickiGarcia, 1970) and Chaetomium globosum (Maret, 1972), but the finding that citrulline occurs in such material is novel. Citrulline normally does not occur in peptide material, but it has been found in proteins of the medulla and inner root sheath cells of hair follicles where it appears to be formed in situ from arginine (Rogers \& Taylor, 1977). In glycopeptides, lysine and arginine are usually involved in $\mathrm{N}$-glycosidic linkages (Kornfeld \& Kornfeld, 1976). Possibly in the R-glucan/chitin complex, arginine is displaced by citrulline. The involvement of lysine and citrulline in a linkage between polysaccharides in the R-glucan/ chitin complex is suggested by the isolation of a fragment (or fragments) containing $(\mathrm{N}$-acetyl)glucosamine and glucose besides lysine and/or citrulline. This fragment originated from chitinase action on a residue from the R-glucan/chitin complex previously digested with exo-( $1 \rightarrow 3)-\beta$-glucanase, a treatment that also removes most of the non-chitinous $N$-acetylglucosamine but none of the amino acids. Therefore this fragment indicates a direct linkage between lysine and citrulline and the chitin chains, possibly through amino groups of (deacetylated) glucosamine residues. This type of linkage has been suggested to occur in chitin/protein complexes of the squid Loligo australis (Hackman \& Goldberg, 1965 ) and in the wall of Mucor mucedo (Datema et al., 1977). However, the results do not exclude other types of linkages. The present finding that, after deacetylation, nitrous acid liberates a component containing glucose and 2,5-anhydromannose indicates that $\mathrm{N}$ acetylglucosamine occurs linked to the anomeric carbon atom. This would explain the liberation of $N$-acetylglucosamine by exo-( $1 \rightarrow 3)-\beta$-glucanase, as noted by others (Troy \& Koffler, 1969), because the enzyme has a limited specificity for residues at the reducing end of the glucan (Nelson et al., 1969).

Taken together, these results suggest that the bridges linking the glucan chains with the chitin (Fig. 4) contain lysine, citrulline, glucose and ( $N$-acetyl)glucosamine, but the sequence and mode of linkage between these monomers remains largely unknown.

We would like to thank Mr C. M. G. Schipma (Laboratory for Developmental Biochemistry, University of Groningen) for performing the amino acid analyses. 


\section{REFERENCES}

Datema, R., Wessels, J. G. H. \& VAN DeN ENDE, H. (1977). The hyphal wall of Mucor mucedo. European Journal of Biochemistry 80, 621-626.

Dygert, S., LI, L. H., Florida, D. \& Thomas, J. A. (1965). Determination of reducing sugar with improved precision. Analytical Biochemistry 13, 367-374.

Fairbain, N. J. (1953). A modified anthrone reagent. Chemistry and Industry 72, 86.

Hackman, R. H. \& Goldberg, M. (1965). Studies on chitin. Australian Journal of Biological Sciences 18, 935-946.

Horton, D. \& Lineback, D. R. (1965). N-Deacetylation; chitosan from chitin. Methods in Carbohydrate Chemistry 5, 403-406.

Horton, D. \& Philips, K. D. (1973). The nitrous acid deamination of glycosides and acetates of 2-amino-2-deoxy-D-glucose. Carbohydrate Research 30, 367-374.

Jeưniaux, C. (1966). Chitinases. Methods in Enzymology 8, 644-650.

Johnson, A. R. (1971). An improved method of hexosamine determination. Analytical Biochemistry 44, 628-635.

Kornfeld, R. D. \& Kornfeld, S. (1976). Comparative aspects of glycoprotein structure. Annual Review of Biochemistry 45, 217-237.

MARET, R. (1972). Chimie et morphologie submicroscopique des parois cellulaires de l'Ascomycète Chaetomium globosum. Archiv für Mikrobiologie 81, 68-90.

Muzzarelli, R. A. A. (1977). Stereochemistry and physical characterization. In Chitin, pp. 45-86. New York: Pergamon Press.

Nelson, T. E., Johnson, J., Jantzen, E. \& KirKwooD, S. (1969). Action pattern and specificity of an exo- $\beta-(1-3)$-D-glucanase from basidiomycetes species QM 806. Journal of Biological Chemistry 244, 5972-5980.

Rogers, G. E. \& TAYloR, L. D. (1977). The enzymatic derivation of citrulline residues from arginine residues in situ during the biosynthesis of hair proteins that are crosslinked by isopeptide bonds. In Protein Crosslinking, part A, pp. 283294. Edited by M. Friedman. London: Plenum Publishing Corporation.

Rosenberger, R. F. (1976). The cell wall. In The Filamentous Fungi, vol. II, pp. 328-344. Edited by J. E. Smith \& D. R. Berry. London: Edward Arnold.

Shively, J.'E. \& Conrad, H. E. (1970). Stoichiometry of the nitrous acid deaminative cleavage of model aminosugar glycosides and glycosaminoglycuronans. Biochemistry 9, 33-43.
Sietsma, J. H. \& Wessels, J. G. H. (1977). Chemical analysis of the hyphal wall of Schizophyllum commune. Biochimica et biophysica acta 496, 225-239.

Sietsma, J. H., Rast, D. \& Wessels, J. G. H. (1977). The effect of carbon dioxide on fruiting and on the degradation of a cell-wall glucan in Schizophyllum commune. Journal of General Microbiology 102, 385-389.

Skujins, J. J., Potgieter, H. J. \& Alexander, M. (1965). Dissolution of fungal cell walls by a Streptomyces chitinase and $\beta$-(1-3)-glucanase. Archives of Biochemistry and Biophysics 111, 358-364.

SPIES, J. R. (1957). Colorimetric procedures for amino acids. Methods in Enzymology 3, 467-477.

StaGG, M. C. \& Feather, M. S. (1973). The characterization of a chitin-associated D-glucan from the cell walls of Aspergillus niger. Biochimica et biophysica acta 320, 64-72.

Troy, F. A. \& Koffler, H. (1969). The chemistry and molecular architecture of the cell walls of Penicillium chrysogenum. Journal of Biological Chemistry 244, 5563-5576.

VAlK, P. VAN DER \& Wessels, J. G. H. (1976). Ultrastructure and localization of wall polymers during regeneration and reversion of protoplasts of Schizophyllum commune. Protoplasma 90, 6587.

VAlK, P. VAN DER, MARchant, R. \& Wessels, J. G. H. (1977). Ultrastructural localization of polysaccharides in the wall and septum of the basidiomycete Schizophyllum commune. Experimental Mycology 1, 69-82.

VRies, O. M. H. DE \& Wessels, J. G. H. (1975). Chemical analysis of cell wall regeneration and reversion of protoplasts from Schizophyllum commune. Archives of Microbiology 102, 209-218.

WANG, M. C. \& Bartnicki-Garcia, S. (1970). Structure and composition of walls of the yeast form of Verticillium alboatrum. Journal of General Microbiology 64, 41-54.

Wessels, J. G. H. (1969). A $\beta$-1,6-glucan glucanohydrolase involved in hydrolysis of cell wall glucan in Schizophyllum commune. Biochimica et biophysica acta 178, 191-193.

Wessels, J. G. H., Kreger, D., Marchant, R., Regensburg, B. A. \& de VRIeS, O. M. H. (1972). Chemical and morphological characterization of the hyphal wall surface of the basidiomycete Schizophyllum commune. Biochimica et biophysica acta 273, 346-358. 